\title{
Technè
}

La science au service de l'histoire de l'art et de la préservation des biens culturels

46 | 2018

Science et conservation

\section{Les triptyques-reliquaires Soltykoff (Paris, Petit Palais) : histoires de collections en restaurations}

The Soltykoff reliquaries in triptych form (Petit Palais, Paris): from collections to restorations

\section{Fabienne Dall'ava}

\section{OpenEdition}

\section{Journals}

Édition électronique

URL : http://journals.openedition.org/techne/312

DOI : $10.4000 /$ techne.312

ISSN : 2534-5168

Éditeur

C2RMF

\section{Édition imprimée}

Date de publication : 1 décembre 2018

Pagination : 11-19

ISBN : 978-2-11-152829-1

ISSN : $1254-7867$

\section{Référence électronique}

Fabienne Dall'ava, « Les triptyques-reliquaires Soltykoff (Paris, Petit Palais) : histoires de collections en restaurations », Technè [En ligne], 46 | 2018, mis en ligne le 19 décembre 2019, consulté le 27 juillet 2020. URL : http://journals.openedition.org/techne/312 ; DOI : https://doi.org/10.4000/techne.312

\section{cc) (†) $\ominus$}

La revue Technè. La science au service de l'histoire de l'art et de la préservation des biens culturels est mise à disposition selon les termes de la Licence Creative Commons Attribution - Pas d'Utilisation Commerciale - Pas de Modification 4.0 International. 
Fabienne Dall'ava

\section{Les triptyques-reliquaires Soltykoff (Paris, Petit Palais) : histoires de collections en restaurations}

The Soltykoff reliquaries in triptych form (Petit Palais, Paris): from collections to restorations

Résumé. Les deux staurothèques mosanes dont traite cet article, conservées au Petit Palais-musée des Beaux-Arts de la Ville de Paris, ont fait partie de la célèbre collection du prince Soltykoff avant d'entrer dans celle des deux frères Dutuit qui les ont léguées à la Ville en 1902. Elles présentaient, depuis la toute fin du siècle dernier, une altération récidivante sous la forme d'une sulfatation vert-bleu. Le démontage partiel de ces ruvres à des fins de traitement a révélé un ensemble de reliques sous l'une des plaques émaillées. Il a également permis d'observer plus précisément les assemblages et les restaurations des triptyques. Pour l'un d'eux (inv. O. Dut 01238), les remaniements ont été mis en æuvre avec le plus grand soin, dans le cadre de son usage cultuel. Au contraire, les restaurations de l'autre reliquaire (inv. O. Dut 01237) témoignent d'une exécution des plus sommaire. Cet examen nous apporte aussi des informations sur l'histoire de ces æuvres entre lieux de culte et collections. Toutefois, l'itinéraire jusqu'au Petit Palais de ces triptyques, dont l'authenticité a été violemment contestée en 1993, demeure mystérieux pour le plus restauré d'entre eux.

Mots-clés. Vente Soltykoff, Collection Dutuit, faux, altération, démontage, reliques.
Abstract. Now in the Petit Palais, Musée des Beaux-Arts de la Ville de Paris, these two reliquaries from the Meuse region were owned by the Russian Prince Soltykoff before entering the collection of the two Dutuit brothers, who bequeathed them to the City of Paris in 1902. Since the end of the last century, they had attested to a recurring alteration in the form of greenish-blue sulphation. Partial dismantling of these works for purposes of treatment revealed a group of relics beneath one of the enamelled plaques. It also enabled us to observe more closely the method of assemblage and the previous restorations of the triptychs. The amendments in one of them had been implemented with the greatest care, with respect to its ritual use. Conversely, the restorations of the other reliquary proved far less diligent. This study also shed more light on the history of these works between their being venerated in places of worship and entering art collections. Their authenticity was, however, strongly contested in 1993, and the long route taken to the Petit Palais by the more carefully restored triptych still remains a mystery.

Keywords. Soltykoff Sale, Dutuit Collection, fakes, alteration, dismantling, relics.

\section{Une collection dispersée}

La vente aux enchères de la célèbre collection réunie par le prince Petr Soltykoff, le 8 avril 1861 et les jours suivants à l'Hôtel Drouot, fut un événement retentissant dans le monde de l'art de l'époque. Tous les amateurs, marchands et musées européens furent au rendez-vous pour tenter d'acquérir les trésors dont le prince se séparait pour d'obscures raisons ou, d'après Eugène Piot, par manque de place dans ses demeures successives ${ }^{1}$. L'intérêt pour les ouvres médiévales, longtemps resté marginal, était maintenant des plus vif et les pièces de cette époque, parmi celles de prédilection du prince, atteignirent des prix d'adjudication élevés à l'issue d'âpres enchères opposant collectionneurs et musées. Les deux staurothèques mosanes (1170-1185) furent adjugées, la première à l'antiquaire parisien Delange, pour 3280 francs sous le numéro 25 du catalogue ${ }^{2}$ (fig. 1) et la seconde, au marchand londonien Durlacher, pour 4350 francs sous le numéro 26 du catalogue $^{3}$ (fig. 2 et voir l'article suivant de J. Durand). Un troisième grand retable d'autel, selon l'appellation du catalogue où il figure sous le numéro 24, pulvérisa ces montants, adjugé pour 7100 francs à un marchand qui le céda ensuite au South Kensington Museum (futur Victoria and Albert Museum $)^{4}$. Ce retable, considéré comme le plus beau et le plus prestigieux des trois exemplaires de la collection Soltykoff, sera malheureusement reconnu plus tard comme un faux.

La dispersion de cette collection insigne, sans publication ni inventaire descriptif, affligeait Alfred Darcel qui regrettait qu'elle n'entre ni au Louvre ni à Cluny ${ }^{5}$, et Eugène Piot déplorait également le départ à l'étranger de bien des chefsd'œuvre, tout en critiquant sévèrement l'indigence du catalogue attribué à Carrand père, conseiller de Petr Soltykoff pour ses acquisitions ${ }^{6}$. Dans le cas de la collection d'Auguste et Eugène Dutuit, cet éparpillement final, si regrettable pour 

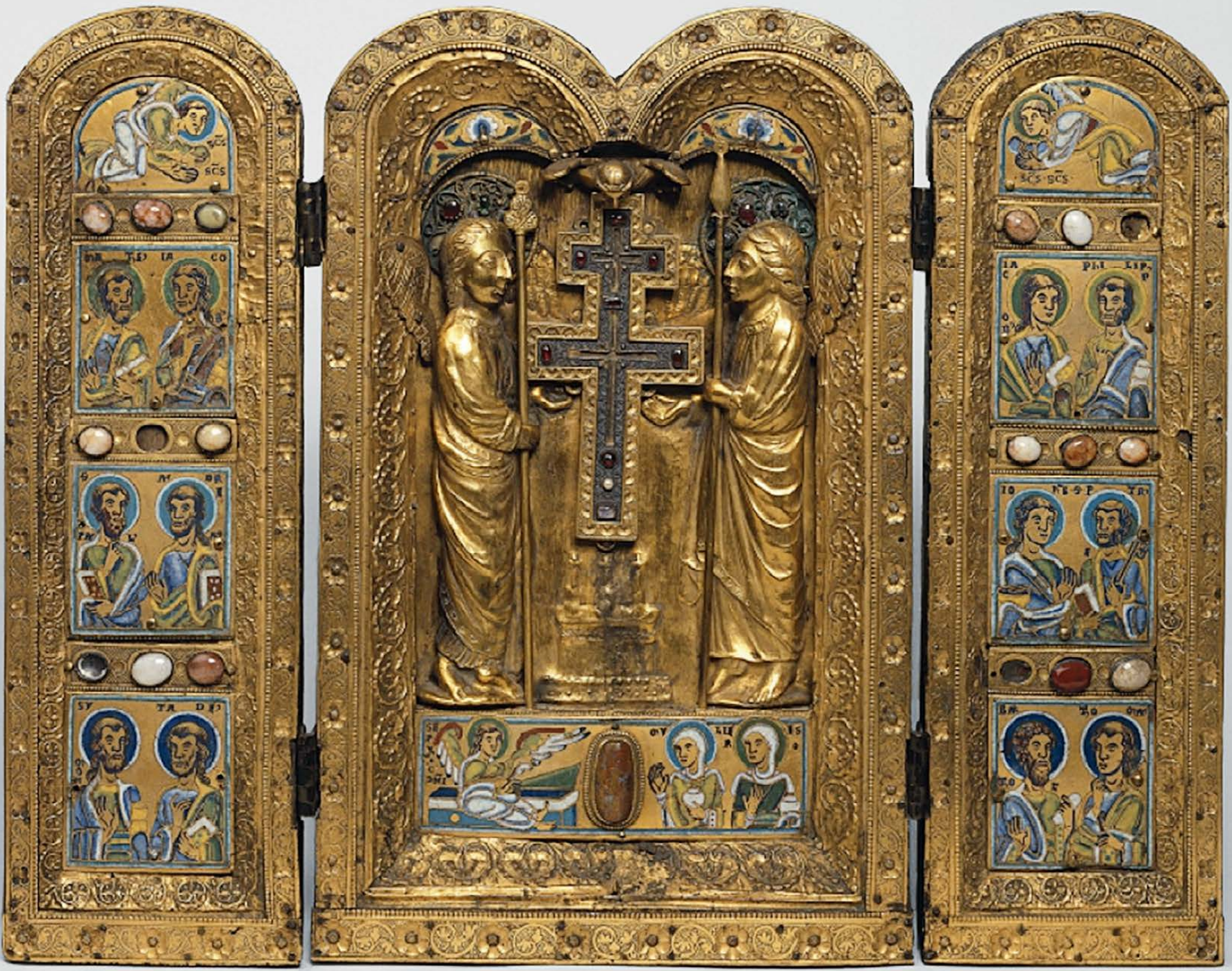

Fig. 1. Le triptyque-reliquaire aux anges dorés, $\mathrm{n}^{\circ} 25$ du catalogue de la vente Soltykoff et inventorié O. Dut 01237. (c) Petit Palais-musée des Beaux-Arts de la Ville de Paris.

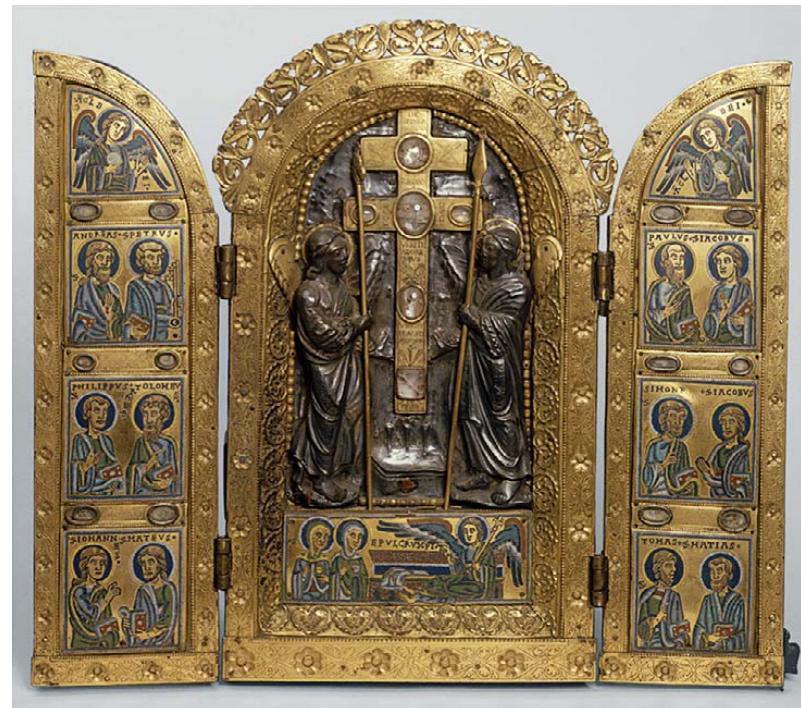

Fig. 2. Le triptyque-reliquaire aux anges d'argent, $\mathrm{n}^{\circ} 26$ du catalogue de la vente Soltykoff et inventorié O. Dut. 01238. (C) Petit Palais-musée des Beaux-Arts de la Ville de Paris. 
d'aussi exceptionnels ensembles, n’a jamais été envisagé. Les deux frères ont acquis, à une date inconnue, les deux reliquaires pour leur futur musée, destiné, dans un premier temps, à l'éducation et à la formation du goût des ouvriers d'art en les familiarisant avec les œuvres des générations passées et, plus tard, à l'accès de tous à la connaissance et aux arts. Ce projet humaniste et généreux, fil conducteur de leurs achats, se concrétisa en 1902 avec le legs à la Ville de Paris de la totalité de leurs collections, riches de quelque vingt mille œuvres parmi les plus belles et les plus emblématiques des époques qu'elles illustrent ${ }^{7}$. Ce legs était accompagné de biens mobiliers et immobiliers qui permettaient de financer la conservation, l'étude et l'accroissement de cet ensemble ${ }^{8}$. Les deux triptyques, après bien des pérégrinations entre lieux de culte et collections, entrèrent alors dans les collections du Petit Palais où ils sont conservés depuis plus d'un siècle ${ }^{9}$.

\section{Une altération récidivante traitée}

C'est à partir de la toute fin $d u x^{\mathrm{e}}$ siècle que ces œuvres ont présenté une dégradation du cuivre se manifestant par la production de sulfates pulvérulents vert-bleu aux emplacements où la dorure était usée. Cette dégradation, bien que traitée ponctuellement à plusieurs reprises, récidivait régulièrement. Cette sulfatation était de toute évidence liée à un ancien traitement à l'acide de ces reliquaires exposés à plusieurs reprises, notamment en 1952 au musée des Arts décoratifs à Paris lors de l'exposition « Trésors d'art de la vallée de la Meuse $^{10}$ »; il est probable que ces présentations publiques aient suscité des nettoyages en vue de parfaire l'aspect des œuvres.

L'étude en laboratoire du C2RMF ${ }^{11}$, rétablissant l'authenticité des émaux des deux triptyques après qu'elle eut été mise en cause ${ }^{12}$, mentionnait également une forte présence de soufre à la surface des plaques émaillées. Cette constatation a permis d'établir que l'acide sulfurique utilisé lors d'un ancien nettoyage était à l'origine de la présence de soufre, sous forme de la couche de sulfures de cuivre noirs au revers des plaques et de la reprise d'altération du cuivre sous forme de sulfates verts lors des variations d'humidité relative ${ }^{13}$.

Pour leur traitement ${ }^{14}$, les deux ouvres ont dû être démontées. Cette opération, à l'automne 2011, a permis d'observer plus précisément les assemblages et les restaurations des triptyques et, ce faisant, de recueillir des précisions sur l'histoire des interventions anciennement menées sur ces œuvres.
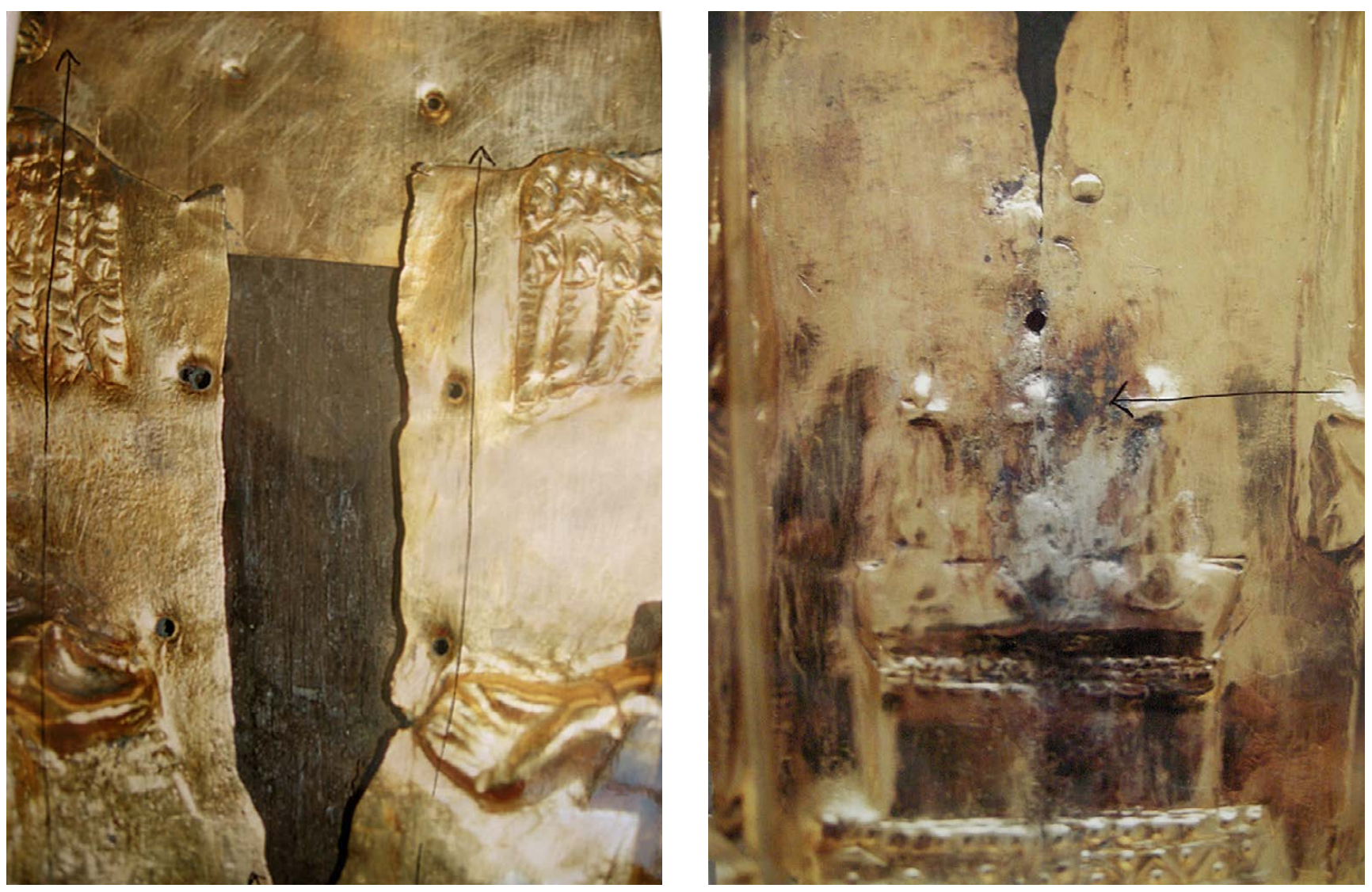

Fig. 3. a. Les plaques des anges du triptyque aux anges dorés (inv. O. Dut 01237) sont découpées irrégulièrement

(flèche de droite). Sous celles-ci, la plaque supérieure plus récente ne présente pas de percement en haut à gauche pour la fixation de la croix ni de trace de soudure pour la fixation des nimbes (flèche de gauche).

b. La ligne de soudure des deux plaques coupe l'autel sur sa verticale. On remarque l'écrasement des reliefs des clous de la Passion et le débordement de métal blanc de soudure sur l'autel (flèche). ( ) F. Dall'ava. 


\section{Le triptyque-reliquaire aux anges de cuivre doré (inv. O. Dut 01237) : une ouvre fortement remaniée}

Le démontage du triptyque-reliquaire aux anges de cuivre doré (fig. 1) a révélé un assemblage de pièces hétérogènes témoignant de remplois et de restaurations anciennes de natures diverses.

Tout d'abord, au centre, la croix à double traverse est fixée suivant un axe légèrement dévié sur la droite. Son démontage révèle que le cadre en bronze doré autour de la petite croix d'argent filigrané, ornée de cabochons montés en bâtes dentelées, a été largement arasé à l'extrémité supérieure de la haste et de chaque côté de ses deux traverses pour supérieure de la croix empiète sur l'aile de l'ange à droite. Ce lourd montage a permis d'adapter au support cette petite croix, probablement réutilisée au vu de sa facture plus raffinée que le cadre. Elle n'a d'ailleurs manifestement jamais été complètement fixée sur ce triptyque. Sept anneaux autour du cadre sont destinés aux clous de fixation; seuls quatre clous subsistent et un des percements pour ces fixations manque sur la plaque, ainsi que sur l'âme en bois du reliquaire.

Sous la croix centrale, les deux plaques de cuivre doré recouvrant l'âme en bois et sur lesquelles se détachent les anges ont subi une lourde intervention : elles ont été échancrées par un découpage irrégulier au-dessus des ailes et se rejoignent en formant une pointe sous la partie inférieure de la haste jusqu'à l'extrémité supérieure de l'autel (fig. 3 a). Elles sont soudées ensemble sur leur ligne médiane et cette trace verticale de l'assemblage est visible au milieu de l'autel dont les reliefs sont écrasés au niveau des clous de la Passion, avec un large débordement du métal blanc de soudure à cet emplacement (fig. 3 b).

Sous l'extrémité supérieure de la plaque des anges se trouve une autre plaque recouvrant l'âme en bois jusqu'aux deux arcs cintrés du sommet. Les lignes de rayures extrêmement régulières à la surface de cette plaque et son

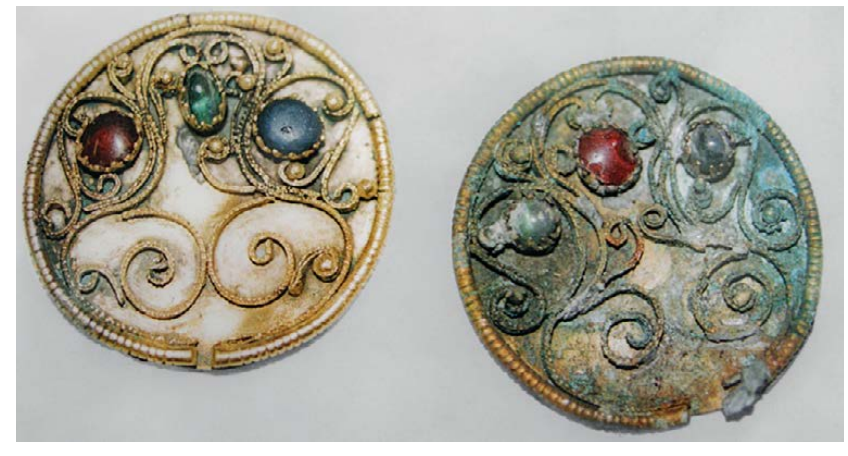

Fig. 4. Les nimbes du triptyque aux anges dorés (inv. O. Dut. 01237), dont les filigranes et les cabochons de verres sont anarchiquement disposés et fixés à l'aide d'une soudure au plomb débordante. Celui de gauche a été nettoyé, celui de droite ne l'est pas encore. ( F. Dall'ava.

épaisseur importante évoquent une facture beaucoup plus récente que celle de la plaque des anges.

L'observation des anges dorés a permis d'étudier aussi la question de leur authenticité. Ils ont été fabriqués selon la technique du repoussé et non de la fonte, comme cela avait été mentionné lors de la remise en cause de leur authenticité $^{15}$. Une ligne d'assemblage de la feuille de métal est discernable de visu sous la main droite de l'ange à gauche et la ligne de soudure de la calotte de l'extrémité de la tête est visible sur l'ange à droite. Il s'agit là d'un procédé traditionnel de réalisation de figures au repoussé, fermées sur le haut de la tête par un disque de métal en forme de calotte, réalisé séparément et assemblé ensuite. L'utilisation de la technique du repoussé ne suffit pas, à elle seule, à établir la contemporanéité des anges avec le triptyque.

Les anges sont recouverts d'une épaisse couche de dorure au mercure quasiment exempte de trace d'usure. Ils peuvent surprendre en raison de leur proportion considérable par rapport à la taille du triptyque et de leur facture sommaire. Certains auteurs ont souligné leurs défauts : « (...) proportions courtaudes, leur grosse tête impassible au nez très fort et aux yeux globuleux $(\ldots)^{16}$. » Pourtant l'argument esthétique ne suffit pas pour mettre en doute l'authenticité de ces anges. Ainsi la comparaison avec certaines figures au repoussé de la châsse de saint Hadelin ${ }^{17}$ tendrait à confirmer leur ancienneté. Rien ne permet, en l'état actuel de nos observations et connaissances, de considérer ces anges comme des faux ${ }^{18}$. À la lumière des observations faites sur l'ensemble des éléments qui composent la partie centrale de ce triptyque, on pourrait envisager l'hypothèse d'un réemploi également pour ces deux figures d'anges.

Les nimbes des anges portent des traces de réfection. Ils ne sont pas fixés à l'aide de petits clous sur la plaque du fond comme cela est l'usage, mais simplement coincés derrière leurs têtes. Ils sont composés d'un disque de tôle sommairement découpé, portant un assemblage chaotique de filigranes et de cabochons de verres rouges, verts et bleus fixés à l'aide d'une soudure au plomb largement débordante (fig. 4). Cette soudure apparaît également sur le revers des nimbes sans qu'on en retrouve la trace sur la plaque sur laquelle ils auraient dû être fixés. Ces nimbes pourraient donc être considérés comme des restitutions utilisant des éléments qui pourraient être plus anciens, filigranes et cabochons de verre.

Le démontage des ailes des anges a révélé, sur la bordure repoussée sur laquelle elles sont fixées, des trous de fixation dont l'écartement ne correspond pas à celui des clous des ailes actuelles. D'autres ailes avaient donc été vraisemblablement fixées à l'origine à cet emplacement.

Sur les deux volets du triptyque, le revers des plaques émaillées porte des traces d'arasement du métal par limage, probablement pour faciliter leur remontage lors d'une restauration. La nuance jaune du métal, surprenante pour du cuivre, censé être quasiment pur et de couleur rouge, 
contraste avec les dépôts noirs de sulfures de cuivre consécutifs au nettoyage à l'acide sulfurique qui a donc précédé cette opération de limage.

La plaque émaillée rectangulaire des Saintes Femmes au tombeau est déformée et soulevée sur sa partie inférieure, témoignant d'une tentative d'ouverture vraisemblablement infructueuse. Sa déformation actuelle rend par ailleurs très difficile le démontage qui n'a pu être réalisé lors de ce traitement ${ }^{19}$.

En conclusion, il apparaît que la partie centrale portant les anges et la croix a été assez lourdement restaurée : la soudure débordante des filigranes anarchiquement disposés sur les nimbes, la découpe irrégulière des plaques des anges, la plaque plus récente sous celles-ci et l'arasement du cadre de la croix centrale témoignent de remaniements importants incorporant des éléments qui pourraient être contemporains de la fabrication du reliquaire (croix filigranée, anges), mais également plus récents (cadre autour de la croix, disque des nimbes et plaque supérieure). Les remontages de ce type ne sont certes pas rares pour les restaurations d'œuvres médiévales lacunaires et endommagées et, bien que difficiles à situer dans le temps, ceux de ce triptyque aux anges dorés pourraient bien ne remonter qu'au XIX ${ }^{\mathrm{e}}$ siècle.

$\mathrm{Au} \mathrm{XIX}^{\mathrm{e}}$ siècle, nombreux étaient les professionnels qui œuvraient en restauration ou qui remaniaient des œuvres d'orfèvrerie, les frontières entre les deux étant peu précises à cette époque. Alexis Berg, à qui l'on a parfois voulu attribuer les remaniements des deux triptyques, est sans doute l'un des plus célèbres ; c'est lui qui aurait « signé », d'une plaque inscrite de son nom et datée de 1854, la fabrication d'un triptyque du Metropolitan Museum of $\mathrm{Art}^{20}$. De précédents auteurs supposaient que les trois reliquaires de la vente Soltykoff avaient séjourné, avant la vente de 1861, dans l'atelier de l'auteur du faux triptyque du Victoria and Albert Museum où nos deux triptyques auraient été également lourdement remaniés ${ }^{21}$. Or toutes les observations des réfections de ce triptyque témoignent au contraire d'un travail bâclé qui ne saurait être l'œuvre d'un maître orfèvre.

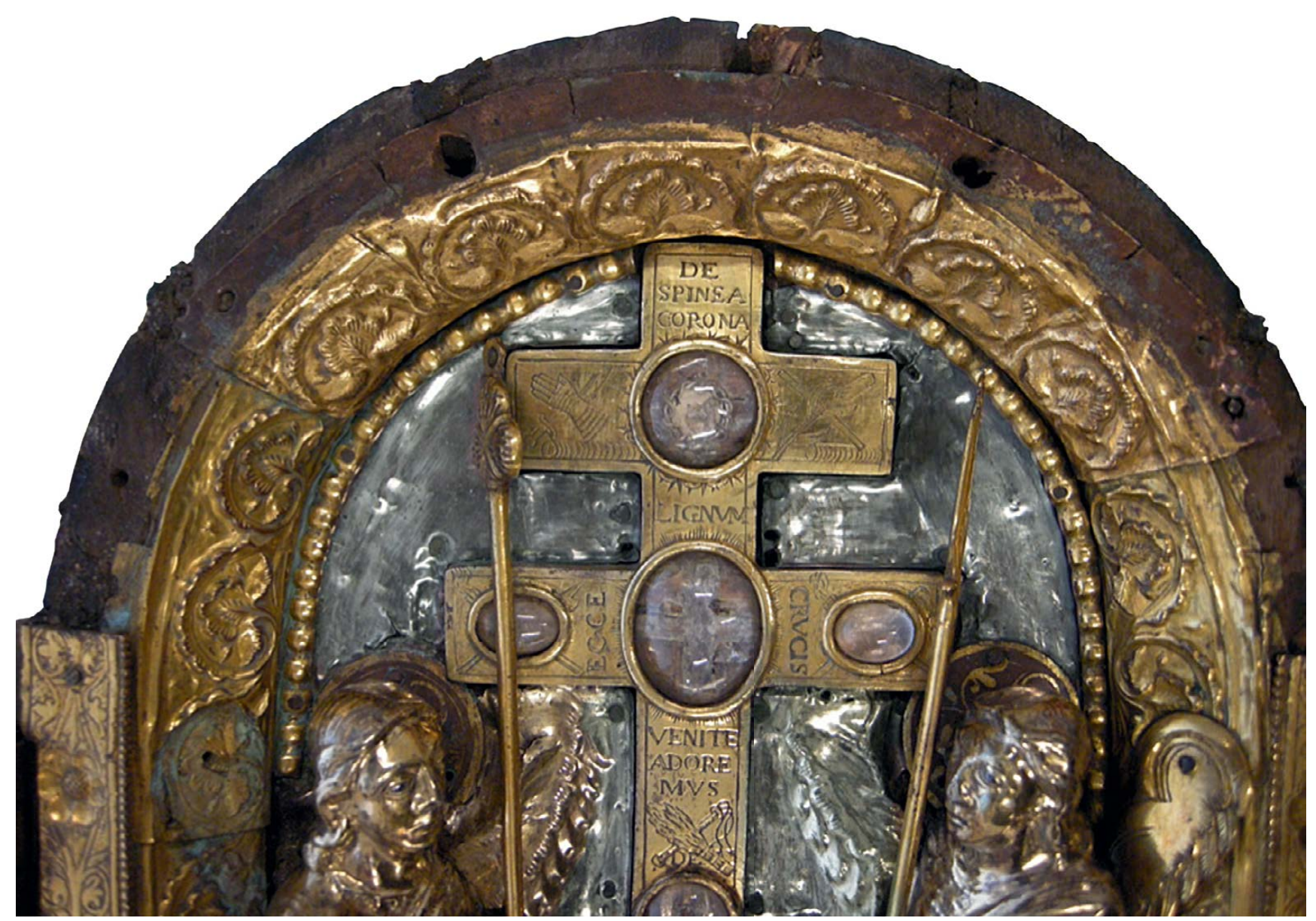

Fig. 5. La croix centrale en argent doré du triptyque aux anges d’argent (inv. O. Dut 01238), portant trois reliques protégées par des cristaux de roche. On remarque le percement unique destiné à la fixation de l'aile de l'ange à gauche, le nielle sur sa pupille et le décor au vernis brun rehaussé d'or des nimbes. @ F. Dall'ava. 


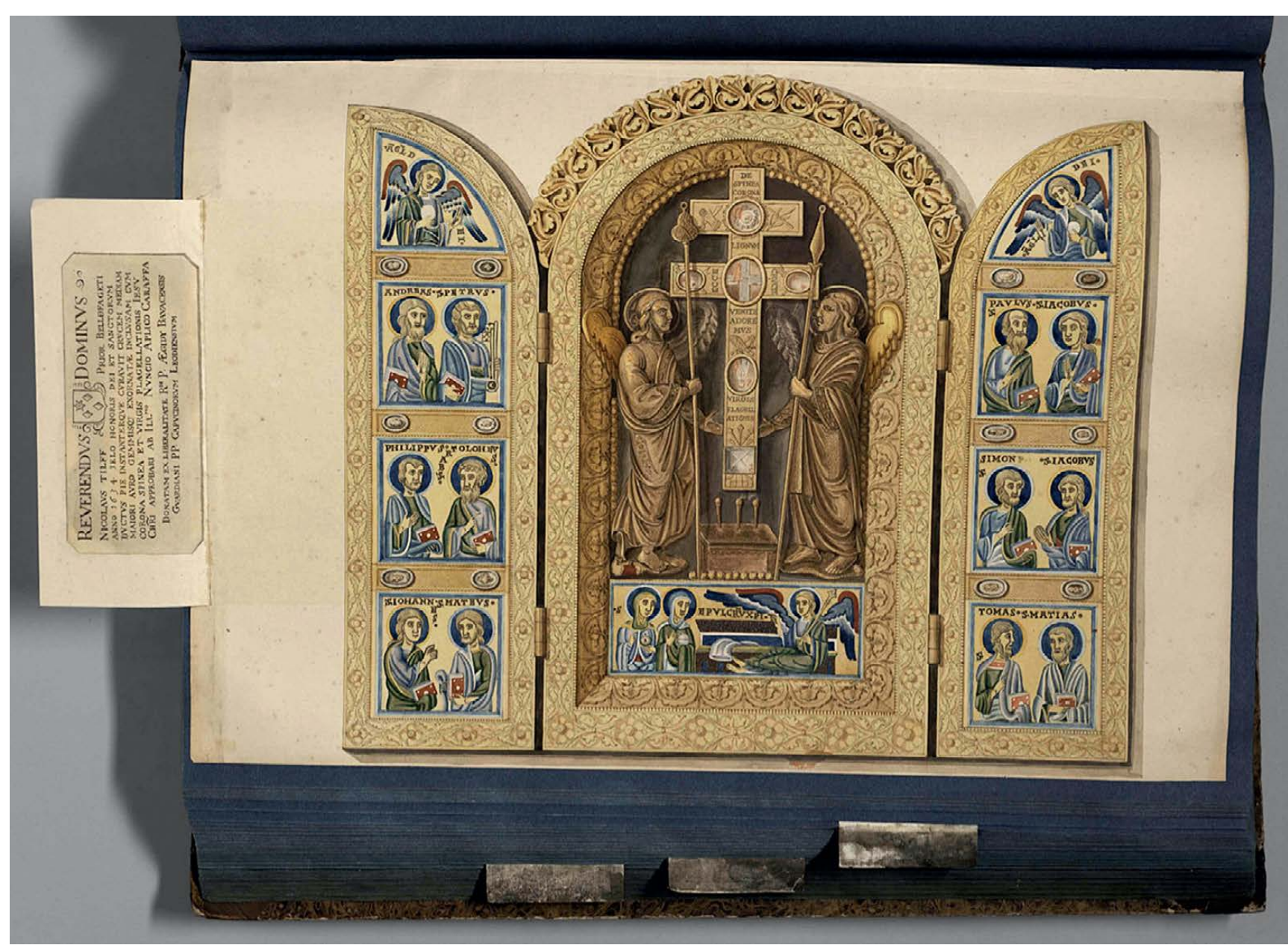

Fig. 6. L'illustration à l'aquarelle du triptyque aux anges d'argent (inv. O. Dut 01238), figurant dans l'ouvrage de 1831 sur la collection du comte de Renesse-Breidbach. L'inscription de la plaque en laiton fixée sur le revers du panneau central est reproduite à gauche.

(c) Natter Fine Arts, Wien. Photo : KHM-Museumsverband.

\section{Le triptyque-reliquaire aux anges d'argent (inv. O. Dut 01238) : un reliquaire remarquablement préservé}

Le triptyque aux anges d'argent (fig. 2 et fig. 5), lui aussi partiellement démonté en vue du traitement de ses plaques émaillées, a conservé un aspect beaucoup plus fidèle à son état originel. Il se distingue aussi par les reliques encore présentes, abritées par la croix centrale et les logettes de ses deux volets.

Les anges d'argent impressionnent par leur exceptionnelle qualité, la perfection de leurs proportions et la grâce de leur attitude. L'utilisation du nielle sur les pupilles accentue la vivacité de leur regard. L'orfèvre a utilisé une unique plaque d'argent très mince tandis que les anges et leurs ailes ont été dorés, ainsi que l'autel. Le ciment de ciseleur, cireux et rouge, destiné à assurer la solidité des reliefs, est visible dans des lacunes sur l'autel et sur le pied de l'ange de gauche. Le démontage des ailes révèle un percement unique sur la bordure où elles sont fixées, indiquant qu'elles sont demeurées à leur emplacement d'origine (fig. 5). Les nimbes de ces anges sont semblables à ceux du reliquaire de la Sainte-Croix de Liège et proviennent peut-être du même atelier ${ }^{22}$. Ils sont enrichis d'un décor au vernis brun rehaussé de feuillages et de volutes dorées, décor admirablement préservé en raison de sa situation en retrait derrière les têtes des anges. On observe aussi que ce vernis brun recouvre les têtes des clous fixant ces nimbes, attestant que ce montage n'a pas été touché depuis la fabrication de l'œuvre. En effet, le vernis brun, obtenu par une fine couche à base d'huile cuite, est particulièrement fragile et l'utilisation d'outils métalliques pour l'extraction des clous l'aurait pour le moins rayé.

Entre les deux anges, la croix à double traverse en argent doré est trop grande pour son emplacement et coupe l'extrémité des mains des anges. Elle a été insérée après la découpe de la tôle du fond puis solidement fixée par de multiples clous, dont certains en fer (fig. 5). Cette croix a donc été placée pour remplacer la croix d'origine lors d'un remaniement, au milieu du tableau central qui, comme nous l'avons constaté précédemment, n'a pas subi d'autres réfections depuis la fabrication de l'œuvre. 
Les inscriptions de cette croix et de la plaque du revers nous apportent de précieuses informations sur l'histoire de ce reliquaire et de ses modifications.

Une inscription, datée de 1634, a été gravée sur une plaque en laiton clouée sur le revers de l'âme en bois du tableau central ; cette inscription a été reproduite sur une aquarelle représentant le reliquaire en 1831 (fig. 6). Elle mentionne une reconnaissance des reliques de la Couronne d'épine et des Verges de la Flagellation par le nonce apostolique Pier Luigi Caraffa (15811655). Les inscriptions de deux logettes à reliques de la face de la croix reprennent scrupuleusement les mentions des reliques gravées sur la plaque du revers. Nicolas de Tilff, prieur de Beaufays et auteur de l'inscription du revers, décrit la croix comme, littéralement, "ornée de plus d'or et de pierres ${ }^{23}$ ». Il s'agissait donc d'une croix non pas en or, mais simplement dorée comme la croix actuelle. La présence de gemmes mentionnée par l'inscription « gemmisque » ne fait pas défaut à cette croix, bien que les reliques soient protégées par de simples cabochons de cristal de roche (fig. 5). Par ailleurs, le style de la croix actuelle et la paléographie de ses inscriptions ne semblent pas plus tardifs que celui de l'inscription du revers, alors que cette croix a été fréquemment attribuée à une restauration du $\mathrm{XIX}^{\mathrm{e}}$ siècle depuis la publication remettant en cause l'authenticité des émaux. Or le remplacement de la croix d'origine par cette croix gemmée et dorée, exécuté dans le plus grand respect de la conservation des reliques et de leurs mentions, pourrait en réalité avoir été réalisé dans le cadre de l'usage cultuel du reliquaire lorsqu'il appartenait au prieuré de Beaufays, près de Liège.

Les volets conservent eux aussi des reliques. Le revers des plaques émaillées (fig. 7), noirci par les sulfures, présente, gravés sous chaque percement ovale, des chiffres romains destinés à repérer l'emplacement exact de chaque logette à reliques lors du démontage. Ces marques ont été gravées lors d'un démontage postérieur à celui effectué lors du nettoyage chimique à l'acide sulfurique, car elles sont de la couleur rouge du cuivre, laquelle contraste avec le dépôt sombre des sulfures du traitement précédent. On observe également un curieux graffito N.N (initiales d'un intervenant ?) sur le revers d'une des plaques supérieures.

Les logettes à reliques sont composées d'un anneau plat et ovale en argent sertissant un verre ; une bande du même métal, munie de dents triangulaires, est soudée autour de l'anneau. Ces logettes sont donc plus tardives et ont été insérées lors d'un remaniement, vraisemblablement en même temps que la croix centrale, dans les percements ovales d'origine des plaques de parement émaillées.

Le démontage de la plaque des Saintes Femmes au Tombeau (fig. 8), toujours à des fins de traitement de la sulfatation des bords et du revers, a révélé un amoncellement de reliques à l'intérieur d'une cavité rectangulaire ménagée dans la partie inférieure de l'âme en bois du reliquaire. Cet ensemble se compose de douze reliques, protégées par une soie bleue (voir l'article de J. Durand dans ce numéro, fig. 2). Les authentiques sur parchemin sont attachés par des liens de nature très diverse en soie, cuir ou coton. Le fond de la cavité est protégé par un rectangle de soie vert vif recouvrant un papier jauni de la même taille.

Les observations faites lors du démontage attestant, entre autres, l'absence de remaniement des figures du tableau central ainsi que la découverte des reliques, sous la plaque émaillée des Saintes Femmes au Tombeau, permettent de conclure que ce second triptyque ne paraît pas avoir été restauré au XIX ${ }^{\mathrm{e}}$ siècle, contrairement au précédent. Quant à la croix, bien que les opinions divergent, une seule réfection en 1634, mentionnée par l'inscription, pourrait être envisagée. La découverte de douze reliques sous la plaque des Saintes Femmes au Tombeau vient confirmer la relative pérennité de l'utilisation pour le culte de ce reliquaire ${ }^{24}$. Toutes les observations faites lors du démontage indiquent clairement qu'il n'y a pas eu de réfections de ce triptyque chez Soltykoff ou pour la vente publique. Ces constatations sont confirmées grâce à la découverte par le Dr Franz Kirchweger, conservateur de la Kunstkammer et

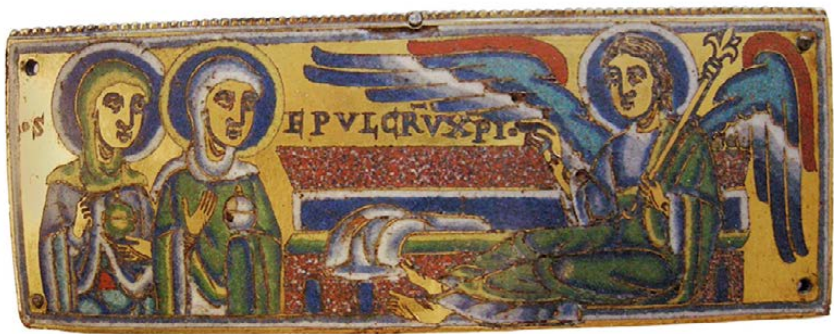

Fig. 8. La plaque des Saintes Femmes au Tombeau du triptyque aux anges d'argent (inv. O. Dut 01238) sous laquelle se trouvaient les reliques. (C) F. Dall'ava. 
Schatzkammer de Vienne, d'une aquarelle (fig. 6) représentant le reliquaire en 1831 - dans un état identique à celui qu'il a actuellement -, dans un recueil inédit des collections du comte Clemens Wenceslaus de Renesse-Breidbach auxquelles il appartenait alors ${ }^{25}$.

Curieusement, ce n'est pas ce dernier triptyque aux anges d'argent, infiniment plus harmonieux et aux figures de plus belle facture que celui aux anges dorés, qui a eu la faveur des éloges et des présentations. Il était déjà décrit comme étant "d'un travail moins soigné peut-être » par Jean-Baptiste Carrand, auteur présumé du catalogue de la vente Soltykoff.

\section{De nouveaux apports à l'histoire des deux triptyques reliquaires}

Si l'itinéraire de ces œuvres jusqu'au Petit Palais garde encore une part d'inconnu, l'histoire du triptyque aux anges d'argent peut être éclairée grâce à l'inscription de 1634, date à laquelle il se trouvait au prieuré de Beaufays, près de Liège, puis grâce à une aquarelle datant de 1831, alors que l'œuvre se trouvait encore dans la même région, dans la collection du comte de Renesse-Breidbach. Après le décès de ce dernier suivi de la vente de la collection en 1836, le reliquaire figure sous le numéro 125 du catalogue de vente ; il est adjugé pour la somme de 700 francs à Fuchs et Debruge-Duménil, qui se porte aussi personnellement acquéreur d'un grand nombre d'œuvres ${ }^{26}$. Sans pouvoir l'affirmer, faute de mentions écrites, on pourrait toutefois supposer que Petr Soltykoff aurait ensuite acheté ce reliquaire - en même temps qu'une grande partie de la collection ${ }^{27}$-, sous seing privé, en 1849 , à la famille de Louis Fidèle Debruge-Dumesnil décédé en 1838. En effet, le triptyque ne figure plus par la suite sur les catalogues de vente du reste des œuvres de la collection. Acquis à la vente Soltykoff par le marchand anglais Henry Durlacher ${ }^{28}$, il serait ensuite passé dans la collection de G. H. Morland ${ }^{29}$, par ailleurs amateur d'émaux, dispersée chez Christie's à Londres le 8 mai 1866. On peut ajouter qu'un des catalogues de la vente Soltykoff porte une note manuscrite mentionnant ce même triptyque comme « revendu le 17 avril 1868, n. $3^{30}$ ». Il s'agit vraisemblablement d'une mention de vente publique mais la date exacte de son entrée dans la collection Dutuit demeure encore inconnue.

En revanche, un mystère quasi total plane sur l'histoire du reliquaire aux anges de cuivre doré dont on ne sait rien avant la vente Soltykoff de 1861, où il est adjugé à l'antiquaire parisien Carle Delange (plutôt qu'au père de celui-ci, Henri), lequel achetait souvent pour le compte des Dutuit ${ }^{31}$. Par ailleurs, il est difficile de dater exactement dans les collections Soltykoff ou Dutuit les traitements de nettoyage à l'acide constatés sur les deux triptyques, les marques sur le revers de leurs plaques attestant au moins deux démontages. De même, on manque d'indications sur les auteurs des précédentes interventions. Faut-il envisager que l'un des démontages et nettoyages des plaques émaillées ait été pratiqué par la maison de restauration André, dont on sait qu'elle a commencé à œuvrer dès la toute fin du XIx ${ }^{\mathrm{e}}$ siècle pour les

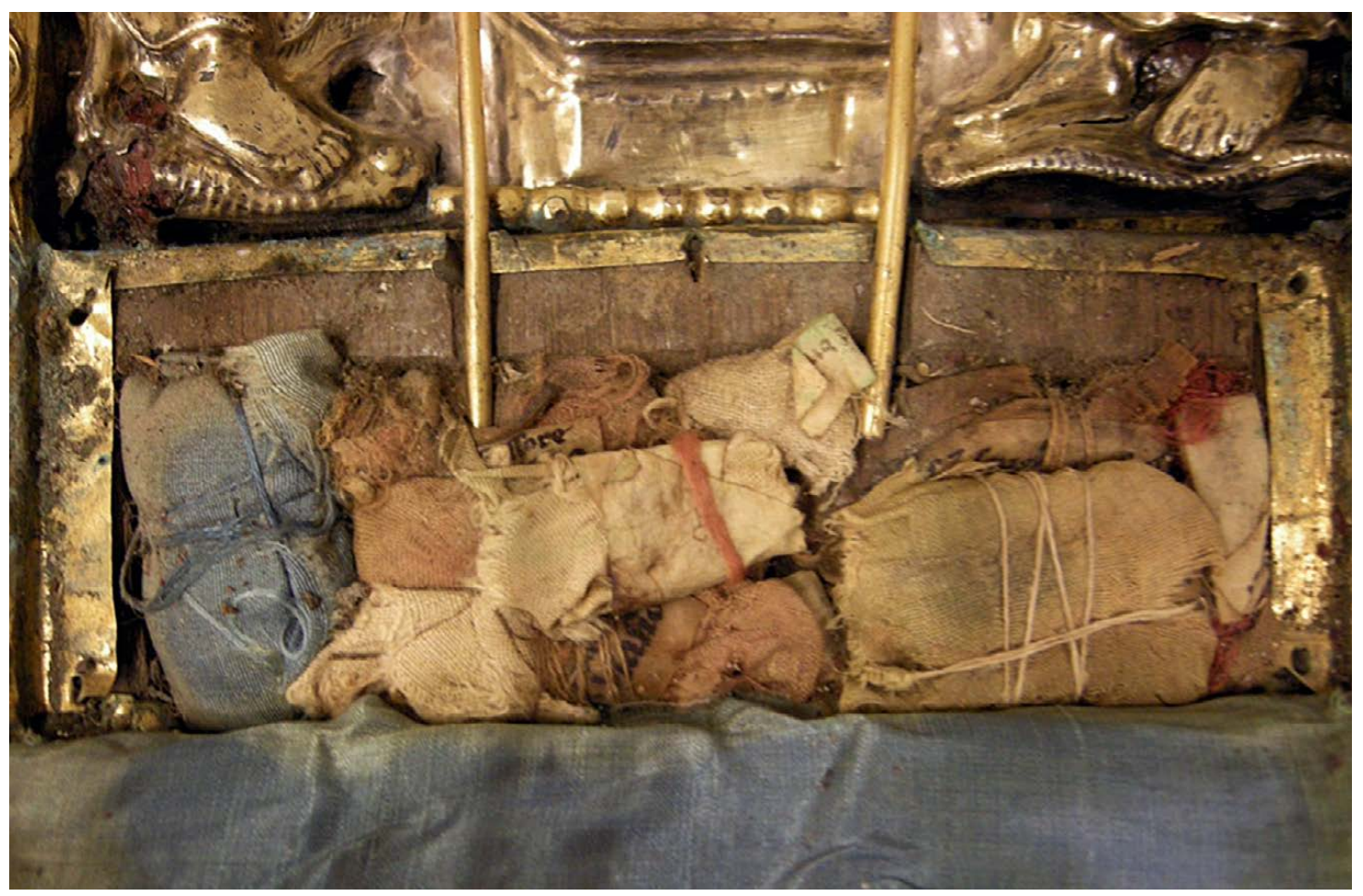

Fig. 9. Les reliques sous la plaque des Saintes Femmes au Tombeau du triptyque aux anges d'argent (inv. O. Dut 01238) sont protégées par une soie bleue dans la cavité creusée dans l'âme en bois. @ F. Dall'ava. 
Dutuit ${ }^{32}$ et qu'elle a opéré, sur un certain nombre d'œuvres médiévales du département des Objets d'art du Louvre, des « révisions » consistant en des démontages et remontages?

Peut-être faudrait-il également déplorer que les deux reliquaires de la collection Dutuit, associés au moment de la vente de la collection Soltykoff à un troisième triptyque, faux, actuellement conservé au V\&A, aient, de toute évidence, injustement pâti de cette proximité avec un pastiche dans la collection du prince.

\section{Remerciements}

Je voudrais remercier, pour l'aide et les informations qu'ils m’ont généreusement apporté : Marie-Cécile Bardoz, Brigitte Bourgeois, Christine Brennan, Dorota Giovannoni, Franz Kirchweger, Patrick Lemasson, Marie Lionnet, José de Los Llanos et Paulette Pelletier-Hornby. Je suis particulièrement reconnaissante à Jannic Durand pour sa relecture attentive et ses précieux avis.
Notes

1. Piot, 1862.

2. Inventorié O. Dut 01237, mentionné dans le texte comme le triptyque aux anges en cuivre doré.

3. Inventorié O. Dut 01238, mentionné dans le texte comme le triptyque aux anges d'argent.

4. Carrand, 1861

5. Darcel, 1861.

6. Piot, 1862.

7. Los Llanos (de), 2013.

8. Pelletier-Hornby, 2010.

9. Patrick Lemasson est actuellement le conservateur en chef, responsable des Objets d'art du Petit Palais-musée des Beaux-Arts de la Ville de Paris.

10. Cat. Exp. Paris, 1952.

11. Biron, Morel, Borel, 1998.

12. Stratford, 1994.

13. Bertholon, 1990

14. Les plaques et les bordures altérées ont été traitées essentiellement par un rinçage contrôlé à l'eau déminéralisée visant à éliminer les restes d'acide et de soufre, suivi d'une déshydratation à l'éthanol.

Une protection à la résine acrylique Paraloid $^{\circledR}$, en très faible concentration dans l'acétate d'éthyle pour éviter toute brillance, a complété ce traitement qui s'est avéré efficace puisqu'aucune récidive n'a été observée depuis.

15. Stratford, 1994, p. 199.

16. Verdier, 1976.

17. Id., ibid.

18. Le métal des anges et des plaques émaillées n'a pas été analysé lors de l'étude du laboratoire du C2RMF, mentionnée note 11 .

19. En revanche, l'écartement de cette plaque est suffisant pour permettre de constater qu'elle ne protège probablement pas de reliques.

20. Inventorié 17.190 .510 et voir Balace, 2009.

21. Stratford, 1994, p. 205.

22. Le reliquaire de la Sainte-Croix de Liège (1160-1180) provenant de l'église Sainte-Croix de Liège est conservé au musée Grand Cursius de Liège.

23. L'inscription en latin est reproduite sur la figure 6 et voir Stratford, 1993.
24. Le reliquaire présente par ailleurs douze autres reliques placées dans les logettes des plaques de parement des volets, ce qui porte en tout le nombre de reliques en place à vingt-sept, avec les trois de la croix.

25. Kirchweger, 2017.

26. Voir Catalogue, 1836

27. Balace, 2009, p. 50.

28. Mention manuscrite des catalogues de vente et Westgarth, 2009.

29. Brennan, 2003

30. Carrand, 1861 , p. 13 , catalogue de la vente Soltykoff annoté par monsieur Batiele, 16 Boulevard Montmartre (probablement marchand de son état). Exemplaire numérisé de Gallica.

31. Mention manuscrite des catalogues de vente et Westgarth, 2009, p. 106.

32. Une lettre du 18 novembre 1899 de F. B Feuardent à Auguste Dutuit l'informe de l'achèvement et de la réussite de la restauration de son Bacchus par André. Ce document a été communiqué par José de Los Llanos à Paulette Pelletier-Hornby qui l'a transmis à l'auteur. Par ailleurs, José de Los Llanos a précisé que les frères Dutuit disaient apprécier les pièces peu restaurées et qu'ils se méfiaient des restaurateurs.

33. Selon Marie-Cécile Bardoz, il s'agit d'une mention des fiches d'œuvres de Serge Grandjean, conservateur du département des Objets d'art du musée du Louvre.

\section{Bibliographie}

Bertholon R., 1990, « Le métal » dans Berducou M. C. (dir.), La conservation en archéologie, Masson, Paris, p. 182-183.

Biron I., Morel D., Borel T., 1998, «Les triptyques reliquaires Dutuit : de l'œil du connaisseur à l'examen en laboratoire, histoire d'une réhabilitation ", Technè n ${ }^{\circ}$ 8, p. 97-106.

Carrand J. B. (auteur présumé), Catalogue des objets d'art et de haute curiosité composant la célèbre collection du prince Soltykoff... Hôtel Drouot... les lundi 8 avril et jours suivants, Paris, 1861, p. 11-13.

Cat. Exp., Paris, 1952, Trésor d'Art de la Vallée de la Meuse. Art mosan et arts anciens du pays de Liège [Exposition, Paris, musée des Arts décoratifs, 1952], Borchgrave d'Altena (de) J., Lejeune M. J. (auteurs des introductions).
Catalogue d'une superbe collection d'antiquités du moyen-âge, objets d'art et curiosités faisant partie du magnifique cabinet délaissé par feu M. le comte Clemens Wenceslas de Renesse-Breidbach... Anvers, 1836, p. 9

Darcel A., 1861, « La collection Soltykoff », Gazette des Beaux-Arts, X, p. 169.

Kirchweger F., 2017, "Gothic and LateMedieval Ivories from the Collection of Clemens Wenceslaus Count of RenesseBreidbach", dans Yvard C. (ed.), Gothic ivory sculpture, content and context, Courtauld Books Online, London, p. 94-98.

Los Llanos (de) J., 2013, « La collection Dutuit, deux frères, un musée ", dans Georgel C. (dir.), Choisir Paris : les grandes donations aux musées de la Ville de Paris. Actes du colloque (Paris, 11-12 octobre 2013), INHA, Paris, p. 1.

Pelletier-Hornby P., 2010, « Les antiques du Musée Dutuit, les grandes collections dans leur siècle ", Compte rendu de l'Académie des Inscriptions et Belles Lettres, vol. 154, n 4, p. 1445-1462.

Piot E., 1863, "Souvenir de quelques collections modernes ", Le cabinet de l'amateur, années 1861 et 1862, p. 158.

Stratford N., 1993, "Un triptyque émaillé mosan du XII ${ }^{\mathrm{e}}$ siècle de Beaufays ", Bulletin de la société royale le vieux-Liège, XII, n 262 , p. 466 et 467.

Stratford N., 1994, "Some 'Mosan' enamel fakes in Paris”, Aachener Kunstblätter 60, p. 199-210.

Verdier P., 1976, « Les staurothèques mosanes et leur iconographie du Jugement dernier ", Cahiers de civilisation médiévale, vol. $16, \mathrm{n}^{\circ} 62$, p. 110 .

Westgarth M. W., 2009, "A biographical dictionary of nineteenth century antique and curiosity dealers", Regional furniture XXIII, Regional furniture society, p. 108.

\section{Documents inédits}

Balace S., 2009, Historiographie de l'art mosan, thèse de doctorat soutenue à l'université de Liège, p. 50, p. 69 et note 194.

Brennan C. E., 2003, "Prince Petr Soltykoff an important nineteenth-century collector of medieval art ", thèse non publiée, vol. II, appendix A, New York, p. 133-134. 